\title{
Motion Compensation by Registration-Based Catheter Tracking
}

\author{
Alexander Brost ${ }^{a}$, Andreas Wimmer ${ }^{a}$, Rui Liao $^{b}$, Joachim Hornegger $^{a}$ \\ and Norbert Strobel ${ }^{c}$ \\ ${ }^{a}$ Pattern Recognition Lab, Friedrich-Alexander-University \\ Erlangen-Nuremberg, Erlangen, Germany \\ ${ }^{b}$ Siemens Corporate Research, Princeton, NJ, USA \\ ${ }^{c}$ Siemens AG, Healthcare Sector, Forchheim, Germany
}

\begin{abstract}
The treatment of atrial fibrillation has gained increasing importance in the field of computer-aided interventions. State-of-the-art treatment involves the electrical isolation of the pulmonary veins attached to the left atrium under fluoroscopic X-ray image guidance. Due to the rather low soft-tissue contrast of X-ray fluoroscopy, the heart is difficult to see. To overcome this problem, overlay images from pre-operative 3-D volumetric data can be used to add anatomical detail. Unfortunately, these overlay images are static at the moment, i.e., they do not move with respiratory and cardiac motion. The lack of motion compensation may impair X-ray based catheter navigation, because the physician could potentially position catheters incorrectly. To improve overlay-based catheter navigation, we present a novel two stage approach for respiratory and cardiac motion compensation. First, a cascade of boosted classifiers is employed to segment a commonly used circumferential mapping catheter which is firmly fixed at the ostium of the pulmonary vein during ablation. Then, a 2-D/2-D model-based registration is applied to track the segmented mapping catheter. Our novel hybrid approach was evaluated on 10 clinical data sets consisting of 498 fluoroscopic monoplane frames. We obtained an average 2-D tracking error of $0.61 \mathrm{~mm}$, with a minimum error of $0.26 \mathrm{~mm}$ and a maximum error of $1.62 \mathrm{~mm}$. These results demonstrate that motion compensation using registration-based catheter tracking is both feasible and accurate. Using this approach, we can only estimate in-plane motion. Fortunately, compensating for this is often sufficient for EP procedures where the motion is governed by breathing.
\end{abstract}

Keywords: Motion Compensation, Catheter Tracking, Atrial Fibrillation Radiofrequency Catheter Ablation, Electrophysiology

\section{INTRODUCTION}

Atrial fibrillation is the most common sustained heart arrhythmia and a leading cause of stroke. It affects about $2 \%$ of the general population. Common treatment options are medication, cardioversion and radio-frequency catheter ablation (RFCA) performed in electrophysiology (EP) labs [1]. The goal of RFCA is to electrically isolate the left atrium (LA) by ablating around the ostia of the attached pulmonary veins (PVs). A successful ablation procedure prevents interfering electrical signals from muscle sleeves around the PVs from disrupting the normal sinus rhythm [2]. The use of overlay images for fluoroscopic guidance improves the quality of the ablation, reduces procedure times, and is helpful for performing linear ablations, e.g., at the left atrium roof [3-6]. Furthermore, critical structures such as the left atrial appendix can be more easily visualized this way. Within EP labs, C-arm X-ray systems are used for catheter guidance.

Send correspondence to Alexander Brost: E-mail: Alexander.Brost@informatik.uni-erlangen.de 
Such systems image the catheters inside a human body very well. Unfortunately, X-ray images do not provide good soft-tissue contrast. Pre-operative soft-tissue 3-D data sets are included to generate overlay images providing additional anatomical information. Such an overlay image can be merged with the 2-D fluoroscopic images to provide additional guidance [3,5]. In other words, the use of overlay images, perspectively rendered from the pre-operative 3-D data sets, makes it possible to augment live 2-D X-ray projections with soft-tissue information [3,7,8]. In general, an initial 2-D/3-D registration step is required to fit volume and projection data. In case of $\mathrm{C}$-arm CT, it is possible to achieve auto-registration if there is no patient movement.

The drawback of the current method is that the volumetric data set does not move synchronously with the fluoroscopic images when the heart and the catheters are affected by respiration and heart beat. Yet taking into account this motion can be crucial [9]. A first approach for 3-D respiratory motion compensation based on catheter tracking was proposed in [10] and further extended in [11-13], but it requires simultaneous bi-plane fluoroscopy. This leads to an increase in X-ray dose for the patient. A 2-D approach was proposed in [14]. Furthermore, the methods in $[10,12,14]$ use a filtering technique to segment the catheter structure. Since it is purely image based, the approach lacks robustness as other structures may be enhanced that do not belong to the desired catheter. The interference from other structures or noise may be less significant when simultaneous biplane fluoroscopy imaging is used for tracking. However, overlapping instruments can seriously affect the robustness of the tracking algorithm when only monoplane fluoroscopy is available, because there is no image-based constraint from a second view. Learning-based algorithms are more robust in terms of suppressing interfering structures that are not of interest [15]. The advantage of learning-based methods vs. filter-based methods was shown in [13]. This difference is further demonstrated in Fig. 1 using a comparison between the segmentation obtained by filtering and classification. In this paper, we present a novel method that relies on mono-plane fluoroscopy only to perform 2-D motion compensation in comparison to $[10,12,13]$ which require simultaneously biplane fluoroscopy. To this end, we track the circumferential mapping catheter by a combination of learning-based catheter classification and model-based 2-D/2-D registration. This catheter is commonly used for PV isolation and can be firmly positioned at the ostium of the PV considered for ablation. An image of this catheter is presented in Fig. 1(a). We use a classifier cascade trained for the circumferential mapping catheter to segment this catheter. This learning-based algorithm avoids enhancing unwanted structures. The classifier is relatively straightforward to train using a comparatively small window size and hence with a constrained variance for detecting catheter feature points instead of a complete circumferential mapping catheter. The complete mapping catheter is then tracked using the detected catheter feature points and a predefined 2-D lasso model. The tracking of this mapping catheter facilitates a local motion estimation at the ablation site. The resulting motion estimate is then used to animate the static fluorosocopic overlay such that it moves synchronously with the tracked catheter and, therefore, compensates for respiratory and cardiac motion. Our approach was tested on 10 clinical data sets.

\section{MATERIALS AND METHODS}

The proposed method comprises three main steps. First, a 2-D model of the circumferential mapping catheter is generated. Second, the catheter of interest is segmented by a classifier cascade. Third, the 2-D model is tracked relying on 2-D/2-D registration to perform motion compensation. Below, these three steps are explained in more detail.

\subsection{Model Generation}

In the first step, the circumferential mapping catheter is extracted in the 2-D X-ray image by manual interaction. A 2-D elliptical model of the mapping catheter is fitted to the manually selected points [16] in the 2-D fluoroscopic images. The manual interaction is used only for the 


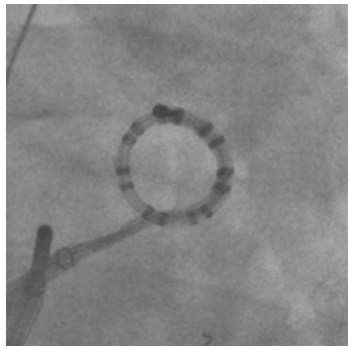

(a) ROI

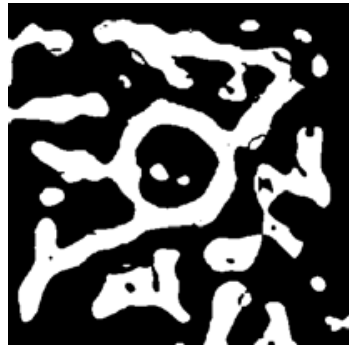

(b) Filter Segmentation

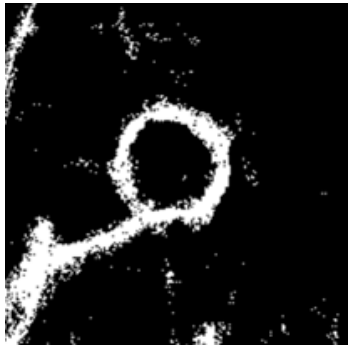

(c) Classification

Figure 1: A region-of-interest of the catheter is presented on the left. The result of the segmentation by filter methods is shown in the middle [10]. It is obvious that there are more false-positive than by segmentation through a boosted classifier cascade as displayed in the image to the right.

first frame of a sequence to generate the 2-D model which is then propagated throughout the remainder of the sequence. In the second step, the catheter is segmented on a region of interest (ROI) restricted to $400 \times 400$ pixels on the original $1024 \times 1024$ image. The position of this region is determined by the center of the tracked catheter model from the previous frame, or from the manual initialization, respectively. This restriction to a smaller search space leads to a faster performance for the subsequent segmentation and registration steps.

\subsection{Catheter Segmentation by Classification}

The catheter segmentation method not only has to be reliable, but it needs to be fast as well. Speed is necessary to ensure that the catheter can be tracked in real-time at the frame rate set at the X-ray acquisition system. We found that a combination of Haar-like features and a cascade of boosted classifiers satisfied both requirements. Haar-like features [17] calculate various patterns of intensity differences. Several feature prototypes are listed in Fig. 2(a). Some features for example detect edges, whereas others focus on line structures. Especially the latter are useful for detecting the catheter, which is a thin, elongated object with a loop at its end, see Fig. 1(a). Actual features are obtained by shifting and scaling the prototypes within a predefined window. In our case, a window size of $15 \times 15$ was found to be sufficient for good results. Thereby, contextual information around the center pixel is considered, which is important to differentiate between catheter and background structures. Even for moderate window sizes, the resulting number of features is large and easily amounts to several hundreds of thousands. Features are calculated efficiently through integral images [17]. To achieve reliable and fast segmentation, the most suitable features for discriminating between catheter and background have to be chosen and integrated into a classifier in a suitable manner. This is carried out by the AdaBoost algorithm [18]. The idea is to combine several weak classifiers, to form a strong classifier. The classifier minimizing the classification error is added to a linear combination of weak classifiers until the overall error is below the desired threshold. After each training iteration, the importance of individual samples is re-weighted to put more emphasis on misclassifications for the next evaluation. Instead of single features and intensity thresholds, we use classification and regression trees (CARTs) [19] as weak classifiers. A CART is a small tree of fixed size. At each node, a threshold $\theta_{j}$ associated with a feature partitions the feature space. Through this decomposition, flexibility is increased and objects with complex feature distributions can be handled. The result of a CART is the value $\alpha_{k}$ of the classifier reached as leave node. An exemplary CART is shown in Fig. 2(b). We organize $N$ strong classifiers $\xi_{i}, \ldots, \xi_{N}$ composed of weighted combinations of CARTs into a cascade, which is illustrated in Fig. 2(c). In our case, $N$ was chosen to be 3 to yield good results. At each stage, a sample is either rejected $(-1)$ or passed on to the next stage. Only if the sample is accepted $(+1)$ at the final stage, it is assumed to belong to the object. Thus during training, the focus is on maintaining a high true positive 


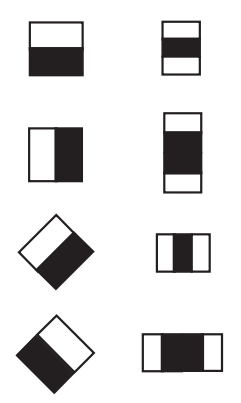

(a) Feature Types

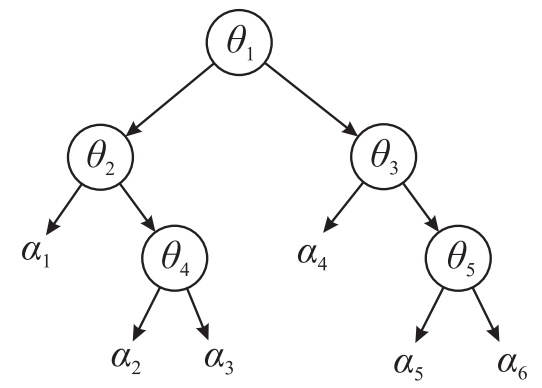

(b) Classification and Regression Tree

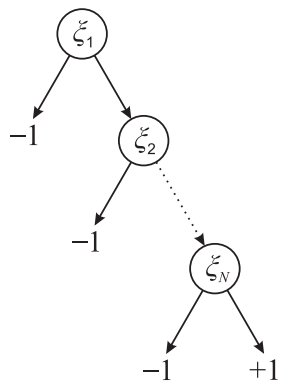

(c) Cascade

Figure 2: Features types and classifier structure for catheter segmentation. (a) Several prototypes of Haar-like features, which are shifted and scaled within a predefined window; intensities within black rectangles are summed up and subtracted from the sums of intensities in white rectangles. (b) Exemplary classification and regression tree (CART) with five feature nodes $\theta_{1}, \ldots, \theta_{5}$ and six leaves $\alpha_{1}, \ldots, \alpha_{6}$. (c) Classifier cascade consisting of $N$ stages with strong classifiers $\xi_{1}, \ldots \xi_{N}$; each strong classifier $\xi_{i}$ consists of a linear combination of weak classifiers, here CARTs.

rate while successively reducing the false positive rate, either by adding more weak classifiers to a stage or by adding an entirely new stage. The training data set consisted of 10 clinical data sets with a total of 498 monoplane frames. For evaluation, the classifier cascade was trained on a leave-one-out basis, i.e., 9 sequences were used for training and the remaining tenth sequence was used for segmentation and tracking.

\subsection{Tracking by Registration}

In the third step, tracking is performed by rigid registration [20] of the catheter model to the segmentation result derived from the previous step. To this end, the same ROI as for the classification is used. As the diameter of the mapping catheter may not be available beforehand or to avoid unnecessary user interaction, the segmented image is thinned as proposed in [21] to generate a skeleton of the catheter. Actual catheters have a diameter between $1.3 \mathrm{~mm}$ and $2.4 \mathrm{~mm}$. Considering a pixel spacing of $0.183 \mathrm{~mm}$ as in our clinical sequences, they are between 7 pixels to 13 pixels wide. Finally, a distance map $\mathbf{I}_{\mathrm{DT}}$ is calculated from the skeleton as proposed in [22]. It encodes the absolute distance from a pixel to its closest segmented catheter pixel. It also provides a smooth representation of the fluoroscopic image with a pronounced minimum around the shape of the mapping catheter to increase the capture range. One pixel $\mathbf{p}$ is accessed by $\mathbf{I}_{\mathrm{DT}}(\mathbf{p})$. The axes of an image are considered as $u$ and $v$. As a 2-D/2-D registration is used, the result is a 2-D pixel offset $\mathbf{t}=\left(\Delta_{u}, \Delta_{v}\right)^{T}$ with $\Delta_{u}, \Delta_{v} \in \mathbb{Z}$. Rotation is not estimated, because 2-D rotation in typical X-ray images taken during EP procedures is usually very small compared to translation. Furthermore, for cases when the lasso catheter is very close to being a circle, the estimation of rotation would require additional landmarks. The average distance between catheter model and segmentation derived from the fluoroscopic image is then considered as the cost value. The optimal translation $\hat{\mathbf{t}}$ is found by minimizing

$$
\hat{\mathbf{t}}=\arg \min _{\mathbf{t}} \sum_{\phi} \mathbf{I}_{\mathrm{DT}}\left(\mathbf{q}_{\phi}+\mathbf{t}\right)
$$

with $\mathbf{q}_{\phi}$ denoting a point on the catheter model and $\phi \in[0,2 \pi]$. As optimization strategy, a nearest neighbor search [23] is used, i.e., the position of the local optimum on a large scale is taken as starting point for the optimization on a smaller scale. The estimated 2-D translation $\hat{\mathbf{t}}$ can be directly applied to the 2-D overlay to move it synchronously with the tracked device. 


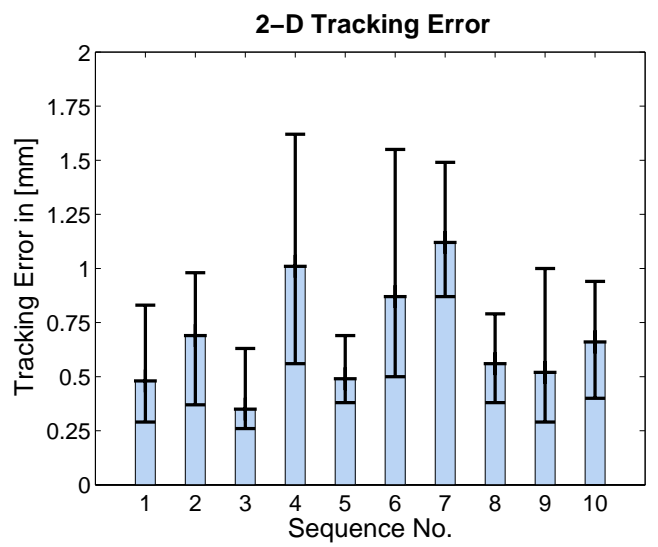

\begin{tabular}{|r|c|c|c|c|}
\hline No. & Mean & Min & Max & Std. \\
\hline 1 & 0.48 & 0.29 & 0.83 & 0.16 \\
\hline 2 & 0.69 & 0.37 & 0.98 & 0.18 \\
\hline 3 & 0.35 & 0.26 & 0.63 & 0.07 \\
\hline 4 & 1.03 & 0.56 & 1.62 & 0.29 \\
\hline 5 & 0.49 & 0.38 & 0.69 & 0.06 \\
\hline 6 & 0.87 & 0.50 & 1.55 & 0.32 \\
\hline 7 & 1.12 & 0.87 & 1.49 & 0.19 \\
\hline 8 & 0.56 & 0.38 & 0.79 & 0.11 \\
\hline 9 & 0.52 & 0.29 & 1.00 & 0.14 \\
\hline 10 & 0.66 & 0.40 & 0.94 & 0.13 \\
\hline $\boldsymbol{\mu}$ & $\mathbf{0 . 6 1}$ & $\mathbf{0 . 2 6}$ & $\mathbf{1 . 6 2}$ & $\mathbf{0 . 2 4}$ \\
\hline
\end{tabular}

Figure 3: Mean tracking error with minimum, maximum, and standard deviation for every clinical sequence. All errors are given in $[\mathrm{mm}]$. The plot to the left was generated from the table to the right. The last row shows the average tracking error over all the 10 sequences as well as the overall minimum and maximum. The total number of frames was 498 . For reference, note that the catheter had a thickness of $2.1 \mathrm{~mm}$. The elliptical shape of the catheter has a diameter between $15 \mathrm{~mm}$ and $25 \mathrm{~mm}$.

\section{EVALUATION AND RESULTS}

For the evaluation of the proposed method, 10 clinical sequences were available. The clinical data was collected at one clinical site and is taken from five different patients. The sequences were acquired during EP procedures on an AXIOM Artis dBC C-arm system (Siemens AG, Forchheim, Germany). Although the data was acquired on a bi-plane system, our motion estimation approach is not restricted to such a system and will work on a mono-plane device as well. As the sequences were acquired during standard EP procedures, our method is evaluated for a typical setup. It involves one circumferential mapping catheter, one ablation catheter and one catheter in the coronary sinus. The tracking error was calculated as the average distance between the motion-compensated catheter model and the manually segmented mapping catheter. Manual segmentation was supervised by a cardiologist. This distance was averaged over all frames of a particular sequence to arrive at an overall tracking error for each sequence. In Fig. 3, we present the mean tracking error together with the minimum and maximum values for each sequence. Over all ten sequences with a total of 498 frames, the mean tracking error was $0.61 \mathrm{~mm}$ with a minimum of $0.26 \mathrm{~mm}$ and a maximum of $1.62 \mathrm{~mm}$. This is small considering that the thickness of circumferential mapping catheters varies between $1.3 \mathrm{~mm}$ and $2.4 \mathrm{~mm}$. For the clinical sequences, a mapping catheter with a thickness of $2.1 \mathrm{~mm}$ had been used. Our method is parallelized for multi-core CPUs and achieves a rate of at least 10 frames-per-second on an Intel Core 2 Duo with $2.20 \mathrm{GHz}$. This is sufficient for clinical applications, as X-ray pulse rates between 1 pulses-per-second (p/s) and $10 \mathrm{p} / \mathrm{s}$ are currently used. An example for one clinical image with and without motion compensation is shown in Fig. 4.

\section{DISCUSSION AND CONCLUSIONS}

We developed a method for respiratory and cardiac motion compensation for use in radiofrequency catheter ablation of atrial fibrillation. This catheter tracking approach involves registration of a catheter model to a catheter segmented by a boosted classifier cascade. The target device for tracking is a circumferential mapping catheter. After manual initialization, the catheter model is tracked throughout the remainder of the sequence. Our evaluation comprising ten clinical data sets yielded an average tracking error of $0.61 \mathrm{~mm}$, with a minimum error of $0.26 \mathrm{~mm}$ and a maximum error of $1.62 \mathrm{~mm}$. From this, we conclude that our method has the potential to significantly improve the accuracy of fluoroscopy overlay techniques for EP navigation. Verification can be achieved only by considering sequences that show the administration 


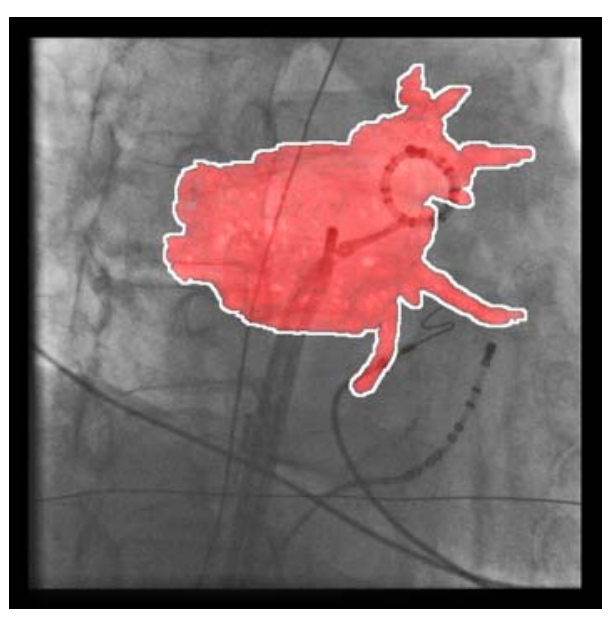

(a)

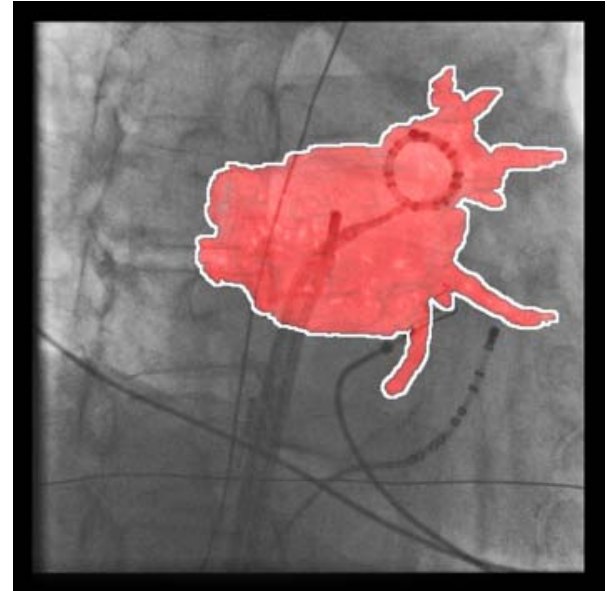

(b)

Figure 4: The current overlay approach involving a static image, rendered from the pre-procedural 3-D data set, is shown on the left. It can be seen that a static overlay may not always exactly align with the current patient anatomy. Please note how the circumferential mapping catheter is starting to move outside the volume, here a segmented left atrium. To the right, the result of our proposed method for motion compensation can be seen. The rendered overlay moves synchronously with the circumferential mapping catheter firmly anchored at the upper left pulmonary vein, and it fits well. The boundary of the overlayed volume is highlighted.

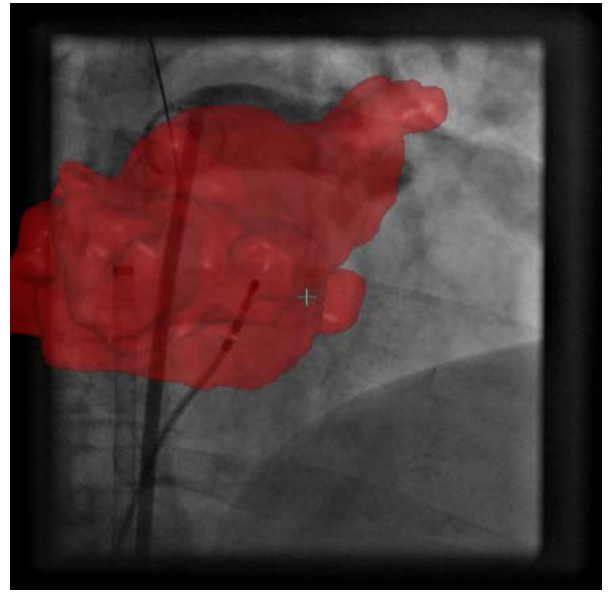

(a)

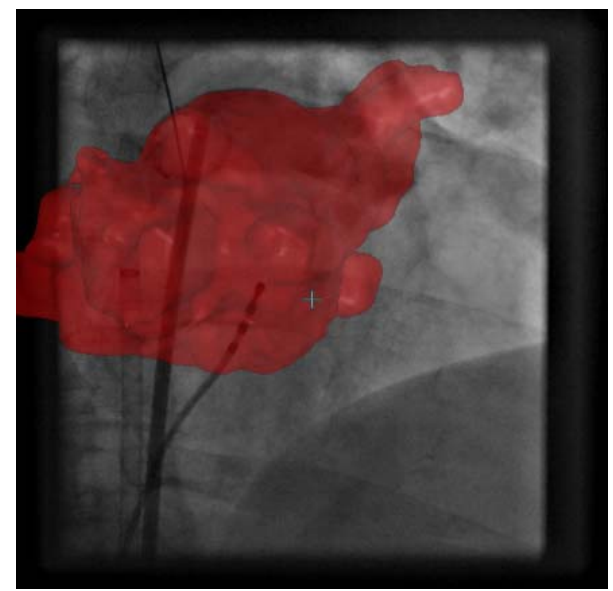

(b)

Figure 5: Administration of contrast agent to outline the left atrium used to verify our motion compensation approach. The image to the left shows a left atrium segmented from a pre-operative MRI overlayed onto a fluoroscopic image without motion compensation. The image to the right shows the same fluoroscopic image, but the overlay image had been moved by our motion compensation approach, resulting in an apparently improved match of the outlines of the left atrium.

of contrast agent. This is a standard step during the procedure to enhance the structure of the left atrium. Figure 5 shows a comparison between a static overlay and a motion compensated overlay for a fluoroscopic sequence involving contrast agent.

The advantages of motion compensation by tracking the mapping catheter that is routinely used during catheter ablation has been demonstrated in [10]. A direct comparison between an overlay with and without motion compensation is presented in Figure 6. However, the pre- 


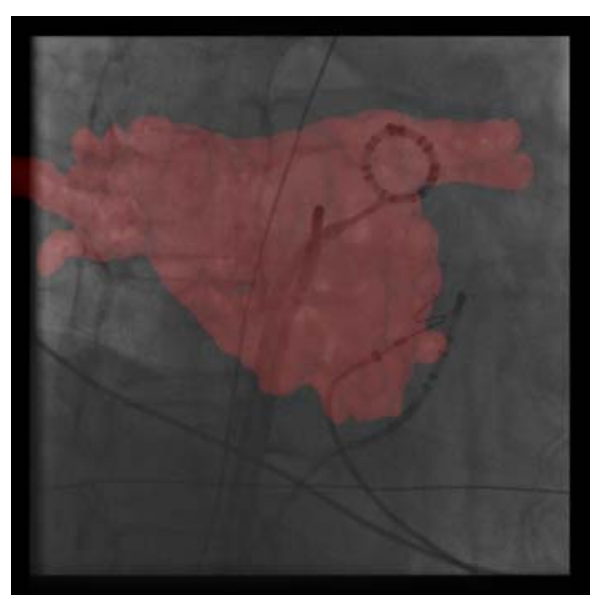

(a) Frame no. 6 without motion compensation

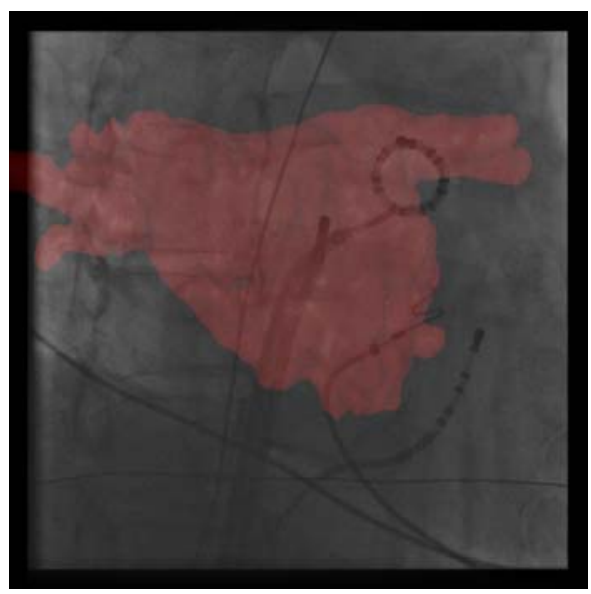

(c) Frame no. 7 without motion compensation

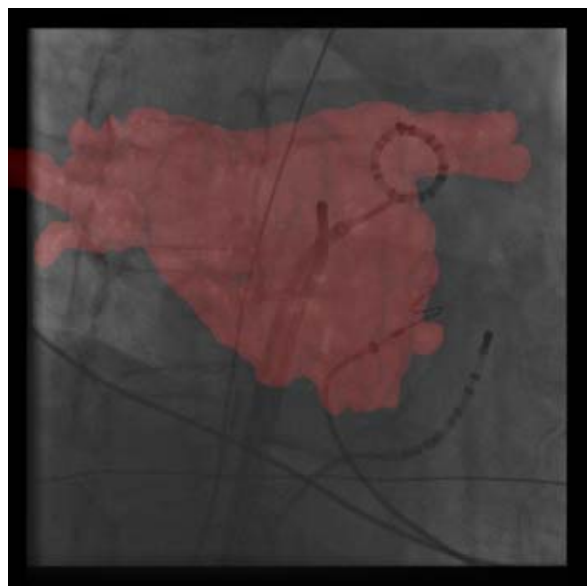

(e) Frame no. 8 without motion compensation

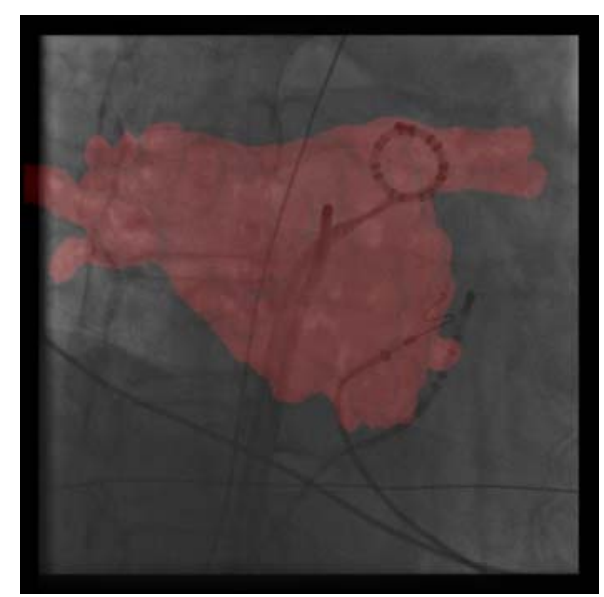

(b) Frame no. 6 with motion compensation

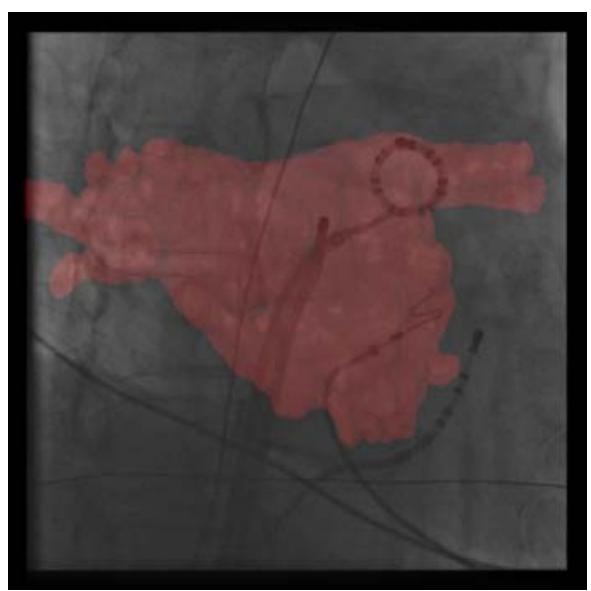

(d) Frame no. 7 with motion compensation

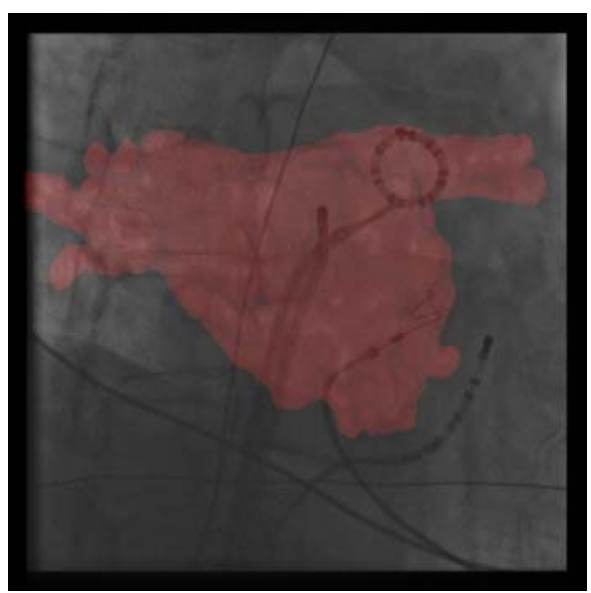

(f) Frame no. 8 with motion compensation

Figure 6: Three successive frames of one of our test sequences visualizes the benefit of a motion compensated overlay. 
vious approach involved 3-D tracking based on bi-plane imaging. In comparison, the newly proposed method tracks this catheter in 2-D monoplane fluoroscopy sequences to reduce the $\mathrm{X}$-ray exposure for both the patient and the physician. Robust and efficient tracking is achieved using a hybrid method involving learning-based catheter classification and model-based registration. We do neither require a complex segmentation approach nor a complex registration algorithm. Instead, we found that the combination of both can also achieve remarkably good results. Furthermore, due to the fact that registration is used, motion estimation and compensation is essentially done in one step.

Due to the slow frame rates during EP procedures, usually 1 to 2 frames-per-second (fps), the previous tracking result is used only to constrain the search region. Our current implementation achieves a frame rate of more than $10 \mathrm{fps}$, on the available data set. As each frame is considered almost independently, our method does not suffer from drift - an often discussed issue for tracking algorithms relying on online updating of the appearance model. Future work might incorporate previous tracking results for prediction. During EP procedures, a rather low dose is used to avoid high X-ray exposures to patients and to physicians. In turn, this leads to a lower image quality in comparison to other applications, e.g., guidewire-tracking in neuroapplications [15]. Therefore, a more sophisticated - in comparison to [24, 25] - but more robust catheter tracking algorithm is required to achieve a good performance. The tip of the ablation catheters used for atrial fibrillation have a diameter between $1.2 \mathrm{~mm}$ and $1.8 \mathrm{~mm}$. Considering a maximum error of $1.62 \mathrm{~mm}$, the overlay would be wrongly translated by the same amount. This would roughly refer to one diameter of the ablation catheter.

The proposed approach does not provide 3-D tracking as depth information is difficult to determine from a single view. Nevertheless, since the motion of the LA can be approximated by a 3-D rigid-body transform [9] and because the LA offers only limited space for a catheter to move about, 2-D motion estimation in the imaging plane may offer an acceptable approximation to arrive at a dynamic overlay. The clinical sequences as well as the gold-standard segmentation will be made publicly available.

\section{Acknowledgements}

This work has been supported by the German Federal Ministry of Education and Research in the context of the initiative Spitzencluster Medical Valley - Europäische Metropolregion Nürnberg. Additional funding was provided by Siemens Healthcare.

\section{References}

[1] Wazni, O., Marrouche, N., Martin, D., Verma, A., Bhargava, M., et al., "Radiofrequency ablation vs antiarrhythmic drugs as first-line treatment of symptomatic atrial fibrillation: a randomized trial," JAMA 293, 2634-2640 (2005).

[2] Calkins, H., Brugada, J., et al., "HRS/EHRA/ECAS Expert Consensus Statement on Catheter and Surgical Ablation of Atrial Fibrillation: Recommendations for Personnel, Policy, Procedures and Follow-Up," Heart Rhythm 4, 1-46 (2007).

[3] Ector, J., De Buck, S., Huybrechts, W., Nuyens, D., et al., "Biplane three-dimensional augmented fluoroscopy as single navigation tool for ablation of atrial fibrillation: Accuracy and clinical value," Heart Rhythm 5, 957-964 (2008).

[4] J. Sra, G. Narayan, D. Krum, A. Malloy, R. Cooley, A. Bhatia, A. Dhala, Z. Blanck, V. Nangia, and M. Akhtar, "Computed tomogrpahy-fluoroscopy image integration-guided catheter ablation of atrial fibrillation," Journal of Cardiovascular Electrophysiology 18, 409-414 (2007).

[5] De Buck, S., Maes, F., Ector, J., Bogaert, J., Dymarkowski, S., et al., "An augmented reality system for patient-specific guidance of cardiac catheter ablation procedures," IEEE Trans. Med. Imaging 24(11), 1512-1524 (2005). 
[6] L. F. Tops and J. J. Bax, "The year in imaging related to electrophysiology," J. Am. Coll. Cardiol. Img. 2(4), 498-510 (2009).

[7] M. Prümmer and J. Hornegger, "Cardiac C-arm CT: a unified framework for motion estimation and dynamic CT," IEEE Trans. Med. Imaging 28, 1836-1849 (2009).

[8] C. Rohkohl, G. Lauritsch, M. Prümmer, and J. Hornegger, "Interventional 4-D Motion Estimation and Reconstruction of Cardiac Vasculature without Motion Periodicity Assumption," in Lect. Notes Comput. Sci, G.-Z. Yang, D. J. Hawkes, D. Rueckert, J. A. Noble, and C. J. Taylor, Eds., 5761, 132-139, (London) (2009).

[9] Ector, J., Buck, S.D., Loeckx, D., Coudyzer, W., et al., "Changes in Left Atrial Anatomy Due to Respiration: Impact on Three-Dimensional Image Integration During Atrial Fibrillation Ablation," J Cardiovasc. Electr. 19(7), 828-834 (2008).

[10] A. Brost, R. Liao, J. Hornegger, and N. Strobel, "3-D Respiratory Motion Compensation during EP Procedures by Image-Based 3-D Lasso Catheter Model Generation and Tracking," in Lect. Notes Comput. Sci., G.-Z. Yang, D. J. Hawkes, D. Rueckert, J. A. Noble, and C. J. Taylor, Eds., 5761, 394-401, (London) (2009).

[11] A. Brost, R. Liao, J. Hornegger, and N. Strobel, "3D model-based catheter tracking for motion compensation in EP procedures," Medical Imaging 2010: Visualization, ImageGuided Procedures, and Modeling 7625(1), 762507, SPIE (2010).

[12] A. Brost, R. Liao, N. Strobel, and J. Hornegger, "Respiratory motion compensation by model-based catheter tracking during EP procedures," Medical Image Analysis 14(5), 695 - 706 (2010). Special Issue on the 12th International Conference on Medical Image Computing and Computer-Assisted Intervention (MICCAI) 2009.

[13] A. Brost, A. Wimmer, R. Liao, J. Hornegger, and N. Strobel, "Catheter Tracking: FilterBased vs. Learning-Based," in Pattern Recognition, M. Goesele, S. Roth, A. Kuijper, B. Schiele, and K. Schindler, Eds., Lecture Notes in Computer Science 6376, 293-302, Springer Berlin / Heidelberg (2010).

[14] A. Brost, R. Liao, J. Hornegger, and N. Strobel, "Model-Based Registration for Motion Compensation during EP Ablation Procedures," in Biomedical Image Registration, B. Fischer, B. Dawant, and C. Lorenz, Eds., Lecture Notes in Computer Science 6204, 234-245, Springer Berlin / Heidelberg (2010).

[15] A. Barbu, V. Athitsos, B. Georgescu, S. Boehm, P. Durlak, and D. Comaniciu, "Hierarchical learning of curves: Application to guidewire localization in fluoroscopy," in CVPR'2007, IEEE Conference on Computer Vision and Pattern Recognition , 1-8, IEEE, IEEE Computer Society, (Los Alamitos, CA, USA) (2007).

[16] R. Halir and J. Flusser, "Numerically Stable Direct Least Squares Fitting Of Ellipses," in WSCG, 253-257 (1998).

[17] P. Viola and M. Jones, "Robust real-time face detection," Int. J. Comput. Vision 57(2), 137-154 (2004).

[18] Y. Freund and R. Schapire, "A decision-theoretic generalization of on-line learning and an application to boosting," JCSS 55(1), 119-139 (1997).

[19] L. Breiman, J. Friedman, R. Olshen, and C. Stone, Classification and Regression Trees, Chapman \& Hall, New York, USA (1984).

[20] D. Hill, P. Batchelor, M. Holden, and D. Hawkes, "Medical image registration," Phys. Med. Biol. 46(3), R1-R45 (2001).

[21] J. Cychosz, "Efficient Binary Image Thinning using Neighborhood Maps," in Graphics Gems IV, 465-473, Academic Press Professional, Inc., San Diego, CA, USA (1994).

[22] H. Breu, J. Gil, D. Kirkpatrick, and M. Werman, "Linear time Euclidean distance transform algorithms," IEEE Trans. Pattern Anal. Mach. Intell. 17, 529-533 (1995).

[23] R. Duda, P. Hart, and D. Stork, Pattern Classification, John Wiley \& Sons, Inc, second ed. (2000). 
[24] S. A. M. Baert, M. A. Viergever, and W. J. Niessen, "Guide wire tracking during endovascular interventions," IEEE Transactions On Medical Imaging 22, 965 - 972 (2003).

[25] D. Palti-Wassermann, A. M. Brukstein, and R. P. Beyar, "Identifying and tracking a guide wire in the coronary arteries during angioplasty from x-ray images," IEEE Transactions on Biomedical Engineering 44, 152-164 (1997). 